\title{
Über eine Darstellung des Periodischen Systems in Kreisform
}

\author{
Von Fritz SCheele* \\ (Z. Naturforschg. 5 a, 11-13 [1950]; eingegangen am 1. August 1949)
}

\begin{abstract}
Es wird ein Periodisches System in Kreisform vorgeschlagen, um die Verhältnisse bei höheren Ordnungszahlen besser überblicken zu können. Die Darstellung führt zu klärenden Einsichten in das Verhältnis von Metallen und Nichtmetallen. Auf den didaktischen Wert der Darstellung wird hingewiesen.
\end{abstract}

$\mathrm{D}$ ie Erweiterung des Periodischen Systems (P.S.) der Elemente über das Uran hinaus und die Charakterisierung der Actiniden als 2. Reihe der Seltenen Erden erfordert einige Revisionen der Darstellung des P.S. in der bisherigen Form. Ein Vorschlag in dieser Richtung wurde kürzlich gemacht ${ }^{1}$. Will man nun einen Überblick über die Verhältnisse bei den Elementen mit höherer Ordnungszahl haben, so wird das rechteckig-tafelförmige System der alten Form unübersichtlich. Es wird daher eine Darstellung in Kreisform vorgeschlagen:

Ausgangspunkt ist das Pauli-Prinzip. Die Kreise von der Mitte her entsprechen den durch die Hauptquantenzahlen $n=1,2,3 \ldots$ festgelegten Energiestufen des Atoms $\mathrm{K}(1), \mathrm{L}(2), \mathrm{M}(3), \mathrm{N}(4), \ldots \ldots$. Die Kreissektoren werden jeweils durch die Nebenquantenzahlen $l=0,1,2,3, \ldots$ mit der üblichen Signatur s (0), p (1), d (2), f(3) bestimmt. Die Reihenfolge der Plätze innerhalb der Sektoren ist festgelegt durch die Magnetquantenzahlen $m_{\tau}$ und die Spinquantenzahlen $-1 / 2$ und $+1 / 2$. Am Kopf der Sektoren, die durch ihre Nebenquantenzahl bezeichnet sind, folgt auf die Gruppennummer des P.S. zunächst die Magnetquantenzahl, dann die Spinquantenzahl (hier dargestellt durch - bzw. + ).

Im Sektor $\mathbf{f}(3)$ ist durch die Bildung einer Triade in der IV. und einer Pentade in der VIII. Gruppe die das P.S. beherrschende Zahl 8 der Gruppen erreicht.

Auf diese Weise wird eine Dreier-Struktur des P.S. gewonnen mit je $3 \times 8$ Untergruppen a, b, c entsprechend der Heranziehung der Nebenquantenzahlen bis $f(3)$. Im Kopf der Sektoren macht eine entsprechende Signatur die Dreier-Struktur sinnfällig.

Diese selbst ist an sich willkürlich. Will man noch den Sektor $\mathrm{g}(4)$ darstellen, so kommt man zu einer Vierer-Struktur mit $4 \times 8$ Untergruppen a, b, c, d (und damit bis zur Ordnungszahl 218). Das alte P.S.

1 F. S c h e e l e, Z. Naturforschg. 4 a, 137 [1949].

Korbach/Waldeck, Flandernweg 8. mit seinen Haupt- und Nebengruppen, deren Bewertung als solche nicht berechtigt ist, läßt sich kreisförmig durch eine Zweier-Struktur mit $2 \times 8$ Untergruppen a, b darstellen. Auch eine Einer-Struktur mit $1 \times 8$ Gruppen ist leicht zu erhalten.

He steht, den es bestimmenden Quantenzahlen entsprechend, unter Be. Um jedoch den gewohnten Zusammenhang mit den übrigen Edelgasen nicht zu verwischen, ist es unter $\mathrm{Ne}$ nocheinmal angedeutet." Um den d-Sektor nicht zu zerreißen, wurde davon abgesehen, La bzw. Ac nach $5 \mathrm{~d}$ bzw. $6 \mathrm{~d}$ zu setzen, wohin diese Elemente der Kenotopie des $4 \mathrm{f}$ - bzw. $5 \mathrm{f}$-Zustandes zu stellen wären.

Im einzelnen liefert die Betrachtung folgende Ergebnisse:

Im ganzen sind nur $2+\Sigma n=23$ Nichtmetalle möglich, von denen das Edelgas 118 noch nicht bekannt ist. Nichtmetalle kommen - abgesehen von $\mathrm{H}$ und $\mathrm{He}-$ nur im p-Sektor vor, d. h. ausschließlich die p-Elektronen können in den Elektronenverband so eingebaut werden, daß das jeweils dadurch hervorgebrachte Element nichtmetallischen Charakter erhält. Mit steigendem Atomgewicht nimmt dieser Charakter von den niedrigeren Ordnungszahlen her schrittweise ab. So entsteht der durch Randschraffur hervorgehobene Zwickel der Nichtmetalle. Sieht man von den in der Chemie der Nichtmetalle weniger bedeutungsvollen Edelgasen ab, so bleiben im p-Sektor 15 Nichtmetalle, die, von dem Supermetalloid F ausgehend, unter stufenweiser Änderung des Charakters nach allen Richtungen im p-Sektor zu den Metallen überleiten. Es entsteht auf diese Weise ein ausgesprochenes Elementenfeld, einerseits gekennzeichnet durch einen besonderen Pol, das F, anderseits bestimmt durch eine Charakteränderung, die schrittweise zum Typus des Metalls führt.

Die Betrachtung der Edelgas-Reihe zeigt, daß Element 118 schon amphoteren Charakter zeigen muß, es liegt innerhalb des Streifens der amphoteren 
Elemente an der Grenzlinie zwischen Metallen und Nichtmetallen.

Die auf den niedrigeren Quantenschalen sich aufbauenden Elemente sind wohlcharakterisierte und deutlich voneinander unterscheidbare „Individuen“, geschieden in Metalle und Nichtmetalle. Auf den edlen Elemente, die wohlcharakterisierten Alkali- und Erdalkalimetalle; im p-Sektor findet man Metalle und Nichtmetalle. In den Sektoren $d(2), f(3)$ usw. folgen dann die Metalle, die jeweils in den Triaden, Pentaden der Edelmetalle ihren kennzeichnenden Schwerpunkt haben.

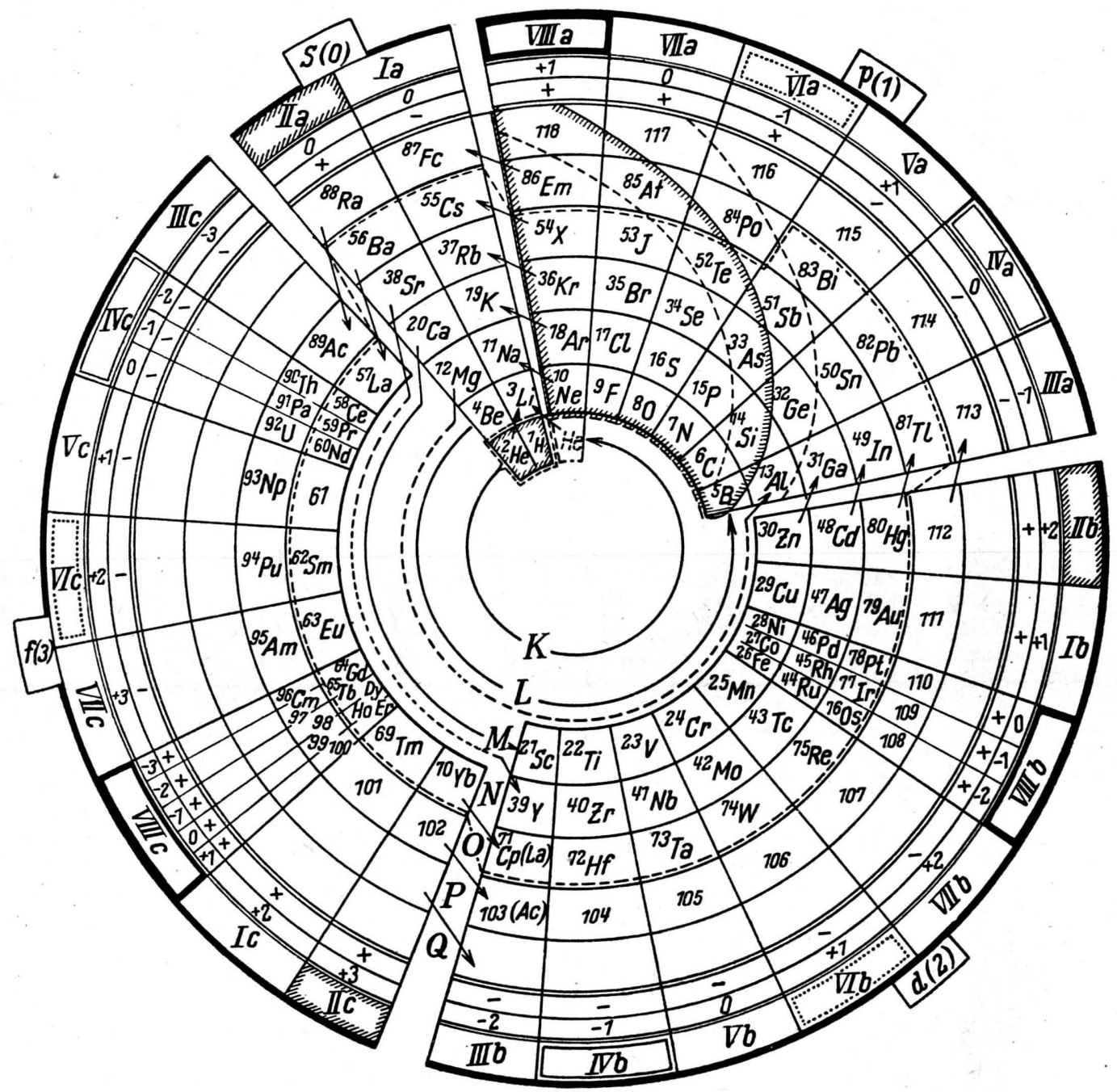

höheren Quantenzuständen tritt der Nichtmetallcharakter mehr und mehr zurück. Die Metalle treten mehr und mehr zu enger verwandten Reihen zusammen, wie sich das zuerst bei den Fe- und PtMetallen, dann aber deutlicher bei den Seltenen Erden zeigt.

Recht deutlich lassen sich nun auch die Unterschiede in der Gruppierung der Metalle erfassen. Im s-Sektor stehen neben den beiden ersten Elementen $\mathrm{H}$ und $\mathrm{He}$, den Grundtypen der unedlen und
Eine punktierte Linie trennt die beständigen Elemente von den radioaktiven (ohne Berücksichtigung der Ausnahmen).

Neben die physikalisch-chemischen Einsichten, die unsere kreisförmige Darstellung vermittelt, tritt ein ausgesprochen didaktischer Wert. Die Abbildung veranschaulicht in einfacher und einleuchtender Weise den Aufbau des Atoms. Die Nahtstellen treten dabei besonders schön hervor: das. Herausspringen der durch die s-Elektronen hervorgebrachten Alkali- und 
Erdalkalimetalle einerseits und der nachholende Ausbau der $3 \mathrm{~d}$-' und $4 \mathrm{~d}$-, $4 \mathrm{f}$ - und $5 \mathrm{f}$-Zustände anderseits. Auf diese Weise wird leicht verständlich, daß ungeachtet der Besetzung der Quantenschalen mit $2 n^{2}=2,8,18,32 \ldots$ Elektronen der Aufbau des P.S. in der alten Form die Perioden 2, 8, 8, 18, 18,
$32,32, \ldots$ aufweisen muß. Dieser Umstand bereitet dem Verständnis erfahrungsgemäß besondere Schwierigkeiten.

Gemäß der Anlage der Darstellung lassen sich die 4 Quantenzahlen für jedes ein Element hervorbringende Elektron ohne große Schwierigkeiten ablesen.

\title{
Der Hall-Effekt von Rubidium
}

\author{
Von Erich Krautz \\ Aus dem Institut für technische Physik der Technischen Hochschule Braunschweig \\ (Z. Naturforschg. 5 a, 13-15 [1950]; eingegangen am 27. Oktober 1949)
}

\begin{abstract}
Es wird die Hall-Konstante von Rubidium zu $10^{6} \cdot A_{\mathrm{H}}=-5920 \pm 140$ [CGS] bestimmt und das Verhalten des Rubidiums hinsichtlich des Leitungsmechanismus im Vergleich mit den übrigen Alkalimetallen diskutiert. Rubidium erscheint als einziger Vertreter der klassischen Elektronengasvorstellung.
\end{abstract}

Für die Erforschung des Leitungsmechanismus im Effekte eine sehr große Bedeutung, da mit ihrer Hilfe Aussagen über Art, Zahl, Bindung und Beweglichkeit der Ladungsträger $\mathrm{zu}$ gewinnen sind. Trotz dieser Wichtigkeit ist die Zahl der kritisch durchgeführten Hall-Effekt-Untersuchungen verhältnismäßig klein und das erfaßte Versuchsmaterial leider noch recht lückenhaft. Grund dafür ist sicherlich die Tatsache, daß der Aufwand für die Durchführung galvanomagnetischer Präzisionsmessungen insbesondere bei chemisch leicht reagierenden Metallen nicht unbeträchtlich ist. Die vom Siandpunkt der Elektronentheorie der Metalle besonders einfachen einwertigen Alkalimetalle oxydieren z. B. sehr rasch an der Luft. Diese experimentelle Schwierigkeit wurde jedoch von verschiedenen Autoren überwunden. W. Nernst und A. v. Ettingshausen ${ }^{1}$ führten als erste HallKonstanten-Messungen an Natrium bei Zimmertemperatur durch, und später A. W. $\mathrm{Smith}^{2}$ an Lithium. In neuerer Zeit wurden recht sorgfältige Messungen an Natrium, Kalium und Cäsium von F. J. S t u d e r und W. D. Will i a $\mathrm{m} \mathrm{s}^{3}$ durchgeführt. Genauere Messungen am Rubidium fehlten indessen bis jetzt.

Auf Grund der experimentellen Untersuchungen von E. $\mathrm{Justi}^{4}$ und einer neu aufgestellten, von speziellen Modellvorstellungen weitgehend unabhän-

1 W. Nernst u. A. v. Ettingshausen, S.-B. Akad. Wiss. Wien, Abt. II a, 94, 560 [1886].

2 A. W. S m ith, Physic. Rev. (2) 8, 79 [1916].

3 F. J. S t ud er u. W. D. Williams, Physic. Rev.

(2) 47, 291 [1935]. gigen Theorie von M. Koh le ${ }^{5}$ haben die galvanomagnetischen Effekte erneut sehr an Interesse gewonnen. Nach diesen Arbèiten ist die von A. S o m m e r $\mathrm{feld}^{\mathbf{B}}$ entwickelte Elektronentheorie, die auf der Vorstellung der Existenz eines freien isotropen Elektronengases beruht, selbst für die elektrisch gut leitenden Alkalimetałle nicht mehr ohne weiteres als gültig anzunehmen, zumindest nicht bei tiefen Temperaturen und sehr starken Magnetfeldern, wo Anisotropien der Bindung und Stoßzeit der Elektronen gefunden wurden.

\section{Die $M$ e $\beta$ probe}

Die größte Schwierigkeit bei der Messung der HallKonstanten von Rubidium lag, wie bei den übrigen Alkalimetallen, zunächst darin, jede Verunreinigung und Oxydation der Meßprobe peinlichst auszuschließen. Diese fallen nämlich deswegen so sehr ins Gewicht, weil man aus meßtechnischen Gründen für Hall-Effekt-Messungen möglichst dünne Metallfolien verwendet, um noch gut meßbare Hall-Spannungen zu erhalten. Bei solchen dünnen Metallfolien machen sich aber Oberflächenverunreinigungen und Oxydschichten bei stark reaktionsfähigen Metallen sehr störend bemerkbar. Man legt in solchen Fällen der Auswertung nämlich eine größere als wirklich vorhandene Dicke der Meßprobe zugrunde und berechnet dann dadurch eine zu kleine Hall-Konstante. Zur Vermeidung solcher Fehlerquellen wurde ein flaches Quarzgefäß benutzt. Es wurde aus zwei plangeschliffenen rechteckigen Quarzplatten $(9 \times 18 \mathrm{~mm})$ hergestellt, die am Rand verschmolzen und mit Zuführungen versehen wurden. Abb. 1 a zeigt die Meßprobe im Maßstab $1: 1,3$. In dieses Gefäß wurde reinstes Rubidium im Vakuum hinein-

4 E. Justi, Ann. Physik (6) 3, 183 [1948].

5 M. K o h le r, Ann. Physik (5) 34, 23 [1939].

${ }^{6}$ A. Sommerfeld, Hdb. Physik 24, II, 333 [1933]. 